\title{
Basic mechanical behaviors and mechanics of shear banding in BMGs
}

\author{
L.H. Dai, Y.L. Bai* \\ State Key Laboratory of Nonlinear Mechanics (LNM), Institute of Mechanics, Chinese Academy of Sciences, Beijing 100080, PR China
}

Received 29 May 2007; accepted 26 October 2007

Available online 23 December 2007

\begin{abstract}
In this paper, some basic mechanical behaviors of bulk metallic glasses (BMGs) were discussed. It can be found from the discussions that the mechanical behaviors of BMGs are mainly due to the formation and operation of shear bands in BMGs. Furthermore, the relevant mechanics of shear banding were investigated in the paper. The theoretical analysis of deformation coupling thermal softening and free volume creation softening demonstrates that the free volume creation and thermal softening can jointly promote the formation of shear bands in BMGs, and the observed post mortem shear band width looks more like that governed by free volume creation.

(C) 2007 Elsevier Ltd. All rights reserved.
\end{abstract}

Keywords: Metallic glass; Shear band; Fracture; Plastic deformation; Free volume

\section{Introduction}

In the 1950s, Turnbull predicted that glass formation in metals is possible if heterogeneous nucleation could be suppressed [1]. Later, Duwez and co-workers fabricated the first metallic glass in the $\mathrm{Au}-\mathrm{Si}$ system with the rapid quenching technique [2]. In the sequent three decades, a number of metallic glasses were produced [3-5]. Prior to the development of bulk metallic glasses (BMGs), very high cooling rates $\left(>10^{5} \mathrm{~K} / \mathrm{s}\right)$ were required to prevent crystallization and the studies of metallic glasses were confined to very thin ribbons or wires. This situation was dramatically changed in the early 1990s when several families of multicomponent alloys, exhibiting a large supercooled liquid region before crystallization, were discovered [6,7], and then metallic glasses formation with a thickness larger than $1 \mathrm{~mm}$ became a common phenomenon. The availability of metallic glasses in the bulk form enables one to extensively study the amorphous microstructure and mechanical behavior of metallic glasses. Since then, attention to BMGs has rapidly increased worldwide [8-13].

The mechanical properties and deformation mechanisms of most conventional metals are well characterized. However,

\footnotetext{
${ }^{*}$ Corresponding author. Tel.: + 861062548133 .

E-mail address: baiyl@lnm.imech.ac.cn (Y.L. Bai).
}

BMGs exhibit behavior unlike those of any other materials. This behavior is not yet fully understood, but we do know, however, that it is related to their composition and atomic structure. Unlike all conventional metals where the atoms are arranged in a well defined and ordered manner, the atoms in BMGs are arranged in randomly arranged patterns with a pronounced short-range order. Such arrangement of the atoms in BMGs results in some unique mechanical properties, such as superior strength and hardness [14], good fracture toughness [15], improved wear resistance [16], etc. However, the lack of significant room-temperature plasticity has limited the application of BMGs as an engineering material so far. Hence, extensive efforts have been made to explore the plastic deformation behavior of BMGs during the past several decades. Spaepen [17] constructed an empirical deformation map and classified the deformation behavior of BMGs into two basic modes: homogeneous and inhomogeneous flows. Homogeneous flow occurs at low stresses and high temperatures. In this type of deformation, each volume element of the specimen contributes to the strain. Inhomogeneous flow usually occurs when a metallic glass is deformed at high stresses and low temperatures (well below the glass transition temperature $T_{\mathrm{g}}$, e.g. room temperature). However, it is this low-temperature inhomogeneous plastic flow of BMGs that we are concerned with, due to the fact that the material usually works under such an environment. The most dominant characteristics of the 
inhomogeneous plastic flow in metallic glasses at high stress and low temperature are that the deformation is highly localized into thin shear bands [18-20]. The formation and interaction of these shear bands of BMGs govern the macroscopic mechanical behavior and give rise to several unconventional deformation features. Firstly, BMGs show certain plasticity in compression but quasi-brittle behavior in tension and simple shear [21-27]. Secondly, BMGs show macroscopic quasi-brittle behavior but with extremely local ductile structures (e.g., dimple, vein-pattern, nanoscale periodicity, etc.) at the nanoscale on fracture surfaces [28-33]. Thirdly, BMGs exhibit no strain hardening, and in compression or indentation, plastic flow is serrated, with many load drops or displacement pop-out [34-42].

In crystalline materials, the formation of shear bands is well known to be attributed to local thermal softening [43-47]. In comparison with crystalline materials, the understanding of shear banding in BMGs is still limited so far. During the past decades, the deformation of localized shear bands and the deformation mechanisms of BMGs have been the subject of theoretical discussion, experimental investigation, and computer simulations. Theoretically, the formation of a shear band in BMGs is attributed to the reduction in the viscosity or the resistance of the material to deformation in a local region, and two hypotheses emerged to explain the local changes in viscosity in shear bands. The first suggests that the reduction of viscosity within shear bands is due to the generation and coalescence of free volume. This idea was originated in the work of Spaepen, who developed a plastic flow model based on a competition between stress-driven creation and diffusion annihilation of free volume [17]. Subsequent works from Argon [47], Stief et al. [48], and Wright et al. [49] also bore this view. Recently, a theoretical framework for inhomogeneous deformation of metallic glasses was proposed and a quasi-static stability analysis was performed by Huang et al. [50]. In this work, the thermal effect and high strain rate cases were not included. The second hypothesis is that local adiabatic heating beyond the glass transition temperature, or even the melting temperature, thus leading to the deformation softening, is the main cause for formation of shear bands in BMGs [51,52]. This idea was stemmed from observing vein-pattern morphologies and melted droplets on fracture surfaces of tested samples. More recently, the coupled effect of the free volume creation softening and the thermal softening on shear banding instability in metallic glasses was discussed by Dai et al. [53]. Moreover, several finitedeformation constitutive frameworks characterizing shear banding in metallic glasses were also developed $[54,55]$.

On the other hand, the deformation and fracture behaviors of BMGs are significantly affected by strain rates. Hence, a number of studies have been made to characterize the mechanical behavior of BMGs under impulsive loadings. Bruck et al. [56] reported that the compressive strength of a $\mathrm{Zr}$-based BMG was independent of strain rates. Similar rate-insensitive behaviors were also observed in Nd-based BMG [39]. Hufnagel et al. [57] observed that the uniaxial compression failure stress of a Zr-based metallic glass decreased with increasing strain rates. Kawamura et al. [58] also reported that the tensile strength of a rapidly solidified $\mathrm{Zr}_{65} \mathrm{Al}_{10} \mathrm{Ni}_{10} \mathrm{Cu}_{15}$ ribbon specimen decreased with increasing strain rates. Strain rate not only affects the fracture strength but also exerts a significant influence on the formation of shear bands in BMGs. Mukai et al. [22] reported that the density of shear bands formed in a Pd-based BMG under tensile loadings increased with strain rates. In our recent studies, this rate dependency of the number density were also observed for $\mathrm{Zr}_{41.2} \mathrm{Ti}_{13.8} \mathrm{Cu}_{12.5} \mathrm{Ni}_{10} \mathrm{Be}_{22.5}$ bulk metallic glass (Vitreloy-1) in shear [24,25]. Conner et al. [59] recently reported some striking findings that $\mathrm{Zr}$-based bulk metallic glass matrix composite exhibited a peculiar "self-sharpening" phenomenon in the course of dynamic penetration. This selfsharpening behavior results from localized adiabatic shear banding a typical dynamic shear instability phenomenon in materials at high strain rates. In addition, several works were carried out recently to focus on the shock compression behavior of BMGs [60-62].

According to the aforementioned observations, one can notice that shear banding in BMGs plays a dominant role in their deformation behavior and macroscopic properties, different from those of conventional metals, especially under impact loading. In this paper, we will firstly compare the phenomena observed by our cooperative groups with others', to identify some basic mechanical behaviors of BMGs. Since these behaviors are closely related to shear bands, in the second part of the paper we will turn to the relevant mechanics of shear banding in BMGs.

\section{Some basic mechanical behaviors}

The unique microstructure in BMGs makes this class of materials exhibit some distinct mechanical behaviors. In particular, it shows some plasticity in compression but quasi-brittle in tension or shear. Actually, it is found that there are macroscopic quasi-brittle but local ductile features at the nanoscale on fracture surfaces, and the load-displacement or stress-strain curves in indentation or compression demonstrate significant pop-ins and serrations. In this section, these behaviors and their correlations will be discussed.

\subsection{Plasticity under compression but quasi-brittle in tension and shear}

BMGs usually exhibit different deformation and fracture behaviors in different loading modes. This character is manifested significantly in the difference in uniaxial tension and compression at room temperature. In tension, BMGs exhibit only elastic deformation behavior, followed by a catastrophical failure without any global plasticity [21-23]. Our experimental results under simple shear showed that the deformation and fracture behavior of BMGs bears the 
same character as that in tension [24,25], as shown in Fig. 1. However, in compression, BMGs usually display a certain plasticity and the deformation behavior is approximately perfectly elastic-plastic with no work hardening

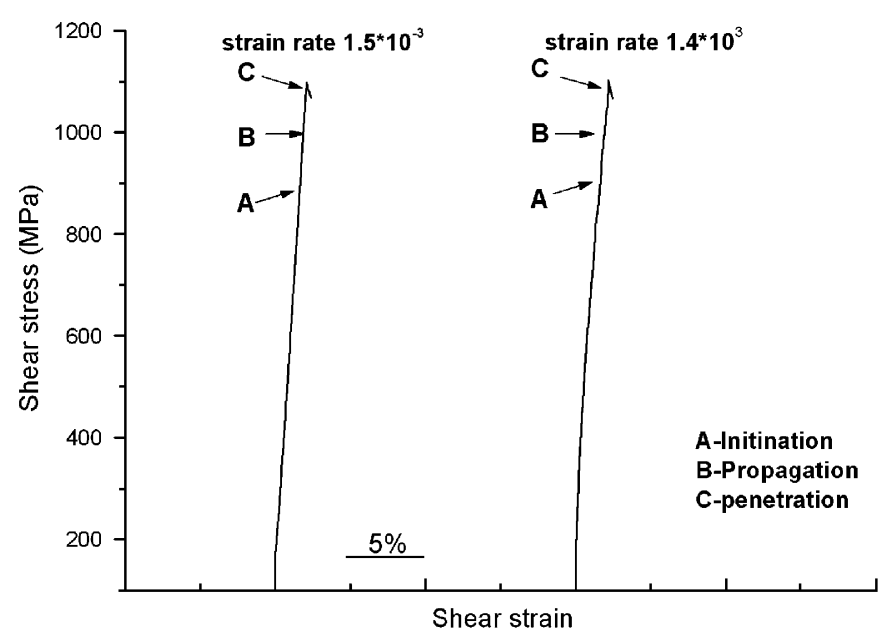

Fig. 1. Shear stress-strain curves of Vitreloy-1 BMG at two strain rates [24].

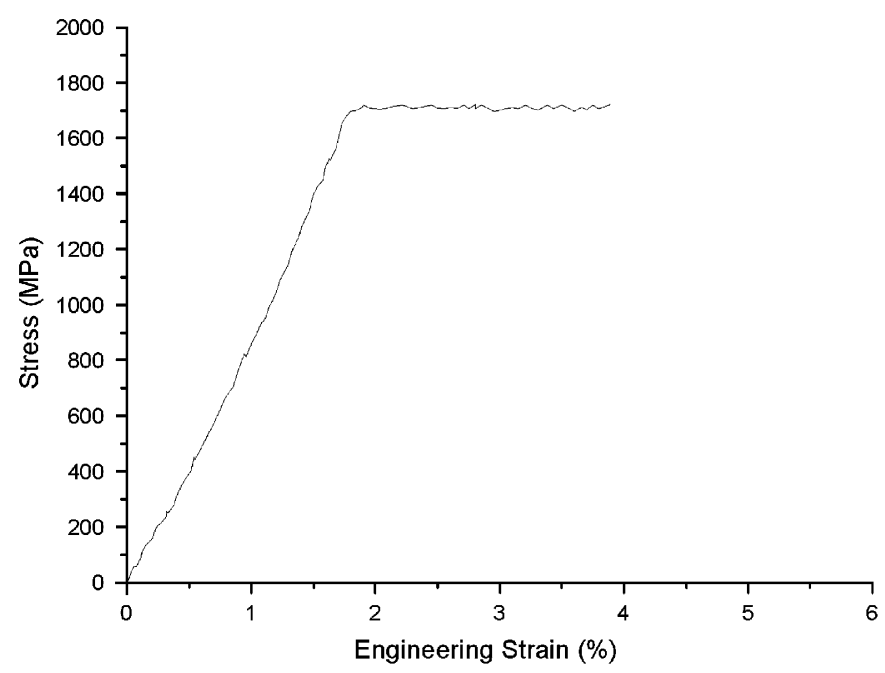

Fig. 2. Uniaxial compression stress-strain curves of Vitreloy-1 BMG [26].
[26,27], which is illustrated in Fig. 2. This difference in the macroscopic mechanical behavior of BMGs should be attributed to the formation and operation of shear bands. In tension, only several isolated shear bands are nucleated. With increasing load, one single dominant shear band propagates rapidly and makes the material fail catastrophically along this main shear band without global plasticity [21]. Quite different from the tension case, multiple shear bands can usually be observed on the sample surface in compression. For example, in our recent compression tests on a $\mathrm{Zr}$-based $\mathrm{BMG}$, multiple shear bands with intersecting, branching, and slipping were observed (Fig. 3a). Also, nanoscale voids can be clearly observed at the intersecting sites of shear bands (Fig. 3b). Obviously, the intersecting, branching, and slipping of multiple shear bands as well as the emerging of nanoscale voids at the intersecting sites of shear bands are thought to be contributed to the observed global plasticity of the material in compression [26]. So, the difference in the macroscopic mechanical behavior of BNGs under compression and shear loadings stems from the difference in their shear banding behavior. It is seen that such a difference in the mechanical behavior of BMGs cannot be observed for most crystalline metals. Hence, plastic in the constrained deformation mode (e.g. compression) but quasi-brittle in the unconstrained mode (e.g. tension or shear) is a peculiar mechanical behavior of BMGs (Fig. 4).

\subsection{Striation and dimple-like patterns of fracture surface}

Flow and fracture behaviors of BMGs depend not only on loading modes as discussed above but also on their chemical composition. From the macroscopic point of view, materials can usually be classified into "ductile" or "brittle" by fracture toughness $\left(K_{C}\right)$. For example, Vitreloy-1 with $K_{C} \sim 80 \mathrm{MPa} \mathrm{m}^{1 / 2}$ is a typical ductile BMG [15], whereas some other BMGs, such as $\mathrm{Mg}$-, Fe-, and Cobased BMGs [28-33], with the toughness $K_{C}$ being about 2-5 MPa ${ }^{1 / 2}$ approaching that of ideal brittle silica glasses $\left(K_{C} \sim 0.68-0.91 \mathrm{MPa} \mathrm{m}^{1 / 2}\right)$, are considered to be brittle. The advent of these materials provides a model material to investigate the dynamic facture of brittle BMGs. During
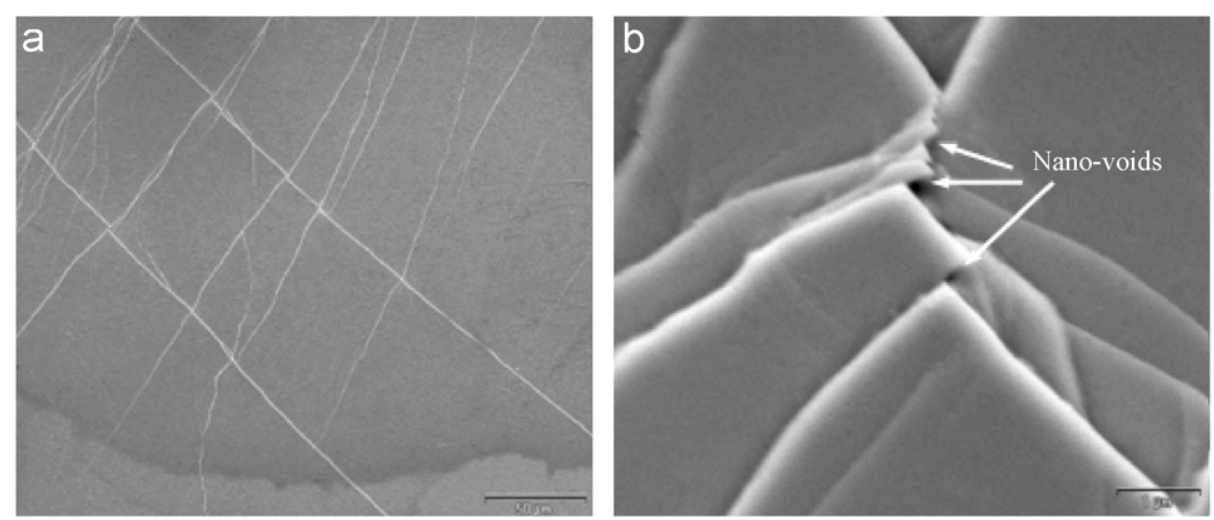

Fig. 3. Multiple shear bands and nanoscale voids at intersecting sites of shear bands [26]. 
a

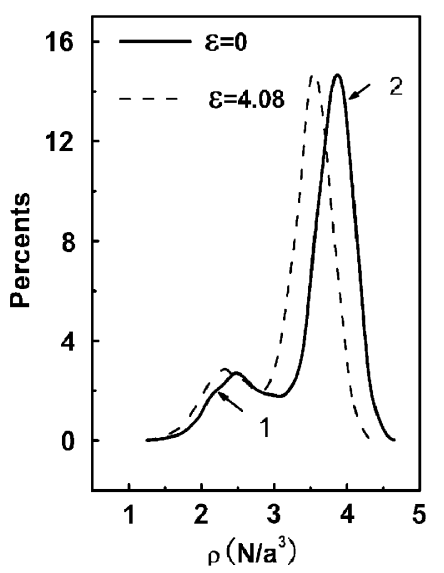

b

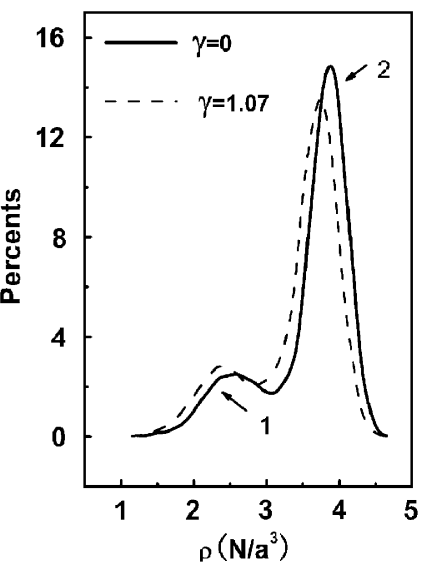

Fig. 4. The deformation-induced density fluctuations: (a) distributions of number density at strain rate $\dot{\varepsilon}=5 \times 10^{9} \mathrm{~s}^{-1}$ and strains $\varepsilon=0$ (solid line) and $\varepsilon=0.0408$ (dash line), respectively, in tension. (b) Distributions of number density at shear strain rate $\varepsilon=4.2 \times 10^{10} \mathrm{~s}^{-1}$ and shear strain $\gamma=0$ (solid line) and $\gamma=1.07$ (dash line), respectively in shear [65].

the past decade, the dynamic fracture of brittle materials has attracted considerable attention. The central issue involved is the dynamic instability of crack tip. It is found that when the crack velocity exceeds a critical velocity $V_{C}$, say $50 \%$ Rayleigh wave velocity, the propagation of the crack tip begins to oscillate and the dynamics of the crack abruptly changes [63]. This dynamic instability was observed in many brittle materials, including plastics, glass, and gels. Fluctuation in the crack tip should result in the release of elastic energy that is transformed into the fracture surface energy and the emission of elastic spreading into the bulk as well as the newly formed fracture surface, which leads to a characteristic pattern sequence known as "mirror", "mist", and "hackle" zones on the fracture surface. Very recently, several studies have demonstrated that the fracture surface of the brittle BMGs also exhibits such a three-zone feature [28-33,67]. For instance, Wang and co-workers [28-30] recently found that the fracture surface of the very brittle $\mathrm{Mg}_{65} \mathrm{Cu}_{25} \mathrm{~Tb}_{10} \mathrm{BMG}$ under three-point bending clearly shows a typical flat mirror zone followed by a mist and shear band branching zone ("hackle" zone). Surprisingly, when the flat mirror zone was carefully examined with high-resolution scanning electron microscope (HRSEM), the 100-nm-scale dimplelike and striation structures are observed, whereas the mirror zone in the other brittle materials is usually featureless. Furthermore, AFM investigation shows that the formation of this dimple structure is due to the nucleation and coalescence of nanovoids [28,29]. The nucleation of nanovoids may be attributed to the fluctuation of free volume or atomic density in the crack tip where the triaxial tensile stress component is very high [64]. Actually, the fluctuation of the density or free volume in BMGs is an intrinsic character of the material under external loading. Our recent molecular dynamics simulation studies also demonstrate that as strain increases to

yield strain the density distribution shifts to the lower part, indicating the creation of new volume during the shear loading process, clearly verifying the occurrence of sheartransformed dilatation in BMGs $[65,66]$, which is shown in Fig. 3. This may imply that the nm-scale dimple structure in the fracture surfaces of the BMG is indicative of the activation of plastic flow processes, possibly as a result of the local softening mechanism. Although this nm-scale dimple structure in the fracture surfaces of the brittle $\mathrm{BMG}$ illustrates significant local plasticity, the contribution of this local plasticity to the global plasticity is very less because the dimple structure is very small. This may be why some BMGs (e.g. Mg-based BMGs) are brittle at the macroscopic scale but ductile at the nanoscale. More recently, an unusual fracture surface pattern was observed in another very brittle $\mathrm{Mg}_{65} \mathrm{Cu}_{25} \mathrm{Gd}_{10}$ BMG by Wang et al. [67]. These authors found an unusual fractographic evolution in this material from nanoscale dimple structure to nanoscale periodic corrugations and then to flat mirror zone along the crack propagation direction (as shown in Fig. 5), which indicates that a ductile to pure brittle transition occurs during the dynamic fracture [67]. They attribute this unusual crack-tip instability to the dependency of the propagation speed on the dynamic
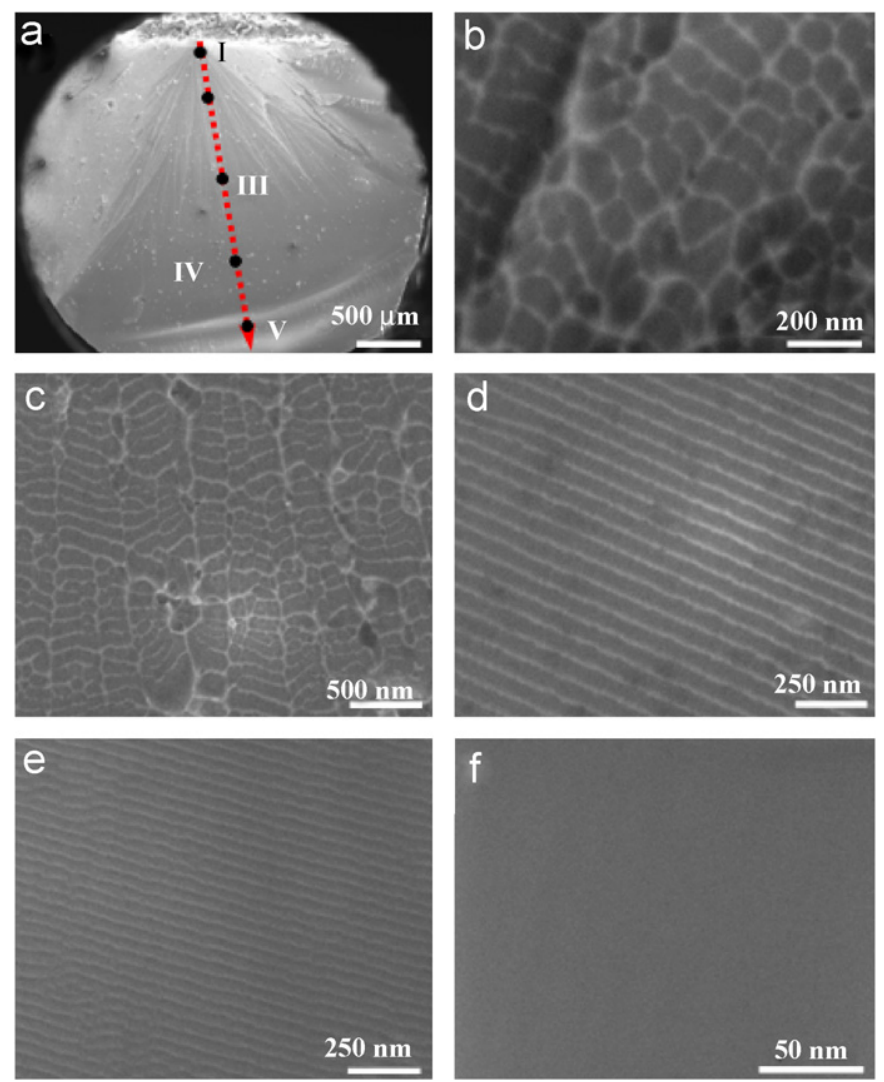

Fig. 5. Fracture surface profile of the BMG bent at the crosshead moving velocity of $1 \mathrm{~mm} / \mathrm{min}$. (a) Overview of the fracture surface (arrow indicates the crack propagation direction). (b) Dimple structure on notch point [point I in (a)]. (c) Image for transition zone on Point II in (a). (d) Periodic corrugation pattern on Point III in (a). (e) Periodic corrugation pattern on Point IV in (a). (e) Mirror zone on point V in (a) [67]. 

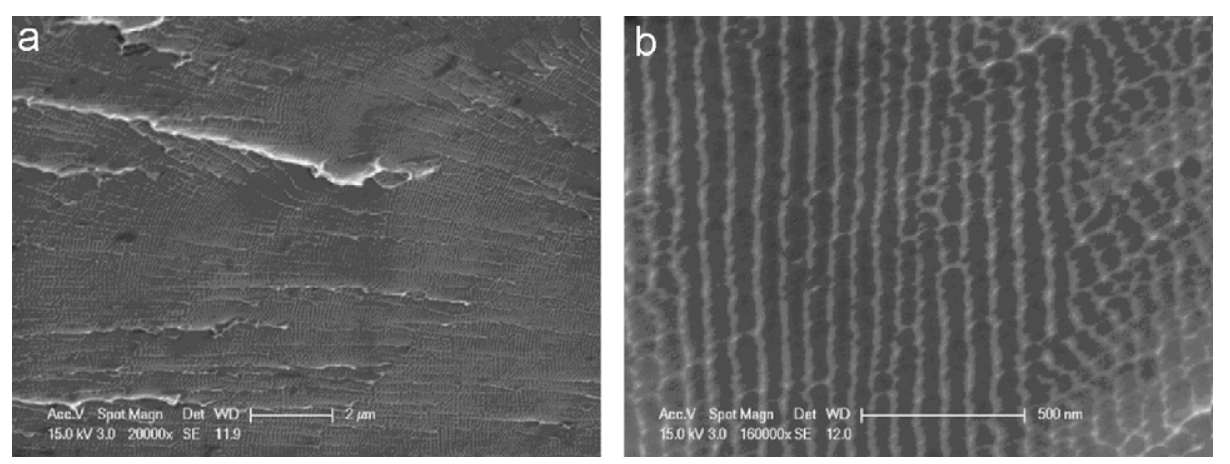

Fig. 6. SEM image of fracture surface of $\mathrm{Zr}_{41.2} \mathrm{Ti}_{13.8} \mathrm{Cu}_{12.5} \mathrm{Ni}_{10} \mathrm{Be}_{22.5} \mathrm{BMG}$ (Vitreloy-1): (a) nm-scale striation and dimple-like structures and (b) higher magnification image of fracture surface showing fine structure of striations and dimples [68].

behavior of viscoelastic-like softening matter in the cracktip front [67].

Surprisingly, our recent experimental investigations have shown that the nm-scale striation and dimple-like structures can also be observed on the fracture surface of very tough Vtitreloy-1 BMG (fracture toughness $\sim 86 \mathrm{MPa} \mathrm{m}^{1 / 2}$ [15]) subjected to high-speed plate impact loadings, as shown in Fig. 6 [68]. Hence, these nm-scale striation and dimple-like structures seem like the intrinsic or universal fracture features for BMGs. However, the physical origin of these unique fracture surface morphological patterns and its correlation with the crack-tip dynamic instability as well as shear banding behavior in BMGs still remain unclear.

\subsection{Nanoindentation behaviors}

The depth-sensing indentation technique has been found to be a powerful tool for investigating material properties such as hardness, elastic modulus, stress-strain response, etc. $[69,70]$. Different from the monotonic loading mode (unaxial compression or tension), the deformation beneath the indenter is in a highly triaxial constrained state. So, the indentation testing can provide a unique way to investigate the deformation behavior of BMGs, especially for those very brittle BMGs, which show no global plasticity in uniaxial tension at room temperature. During the past several years, growing efforts have been made to use this technique to study the deformation behavior of BMGs [35-38,40]. Our results of nanoindentation tests show that the indentation behavior of BMGs exhibits two significant features: the serrated loadingdisplacement curves ( $p-h$ curves) with many distinct displacement bursts and the material pile-up around the indenter, as shown in Fig. 7 [71]. The serrated flow is a typical unstable plastic instability phenomenon, and is not unique to BMGs. In crystalline materials, it can be observed as the Portevin-Le Chatelier (PLC) effect and is associated with dynamic strain aging (DSA) caused by the interaction of moving dislocations with mobile impure atoms [72]. Due to the lack of the line or planar defects (such as dislocations or grain boundary in crystalline

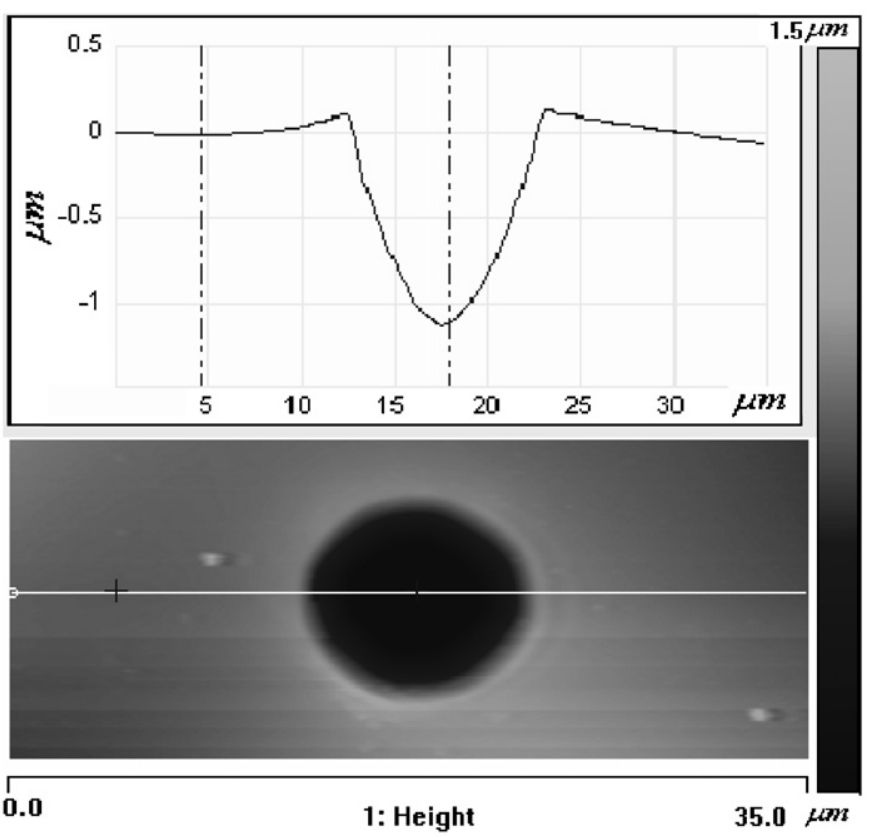

Fig. 7. AFM image of residual indent of Vitreloy-1 in spherical indentation [71].

materials), the serrated flow in BMGs is surely a direct consequence of shear banding.

Extensive investigations demonstrated that the serrated flow in BMGs is highly dependent on strain rates and temperature. Schuh and Nieh [36] reported that the serrated flow in Pd- and Be-free Zr-based BMGs is more significant at relatively lower strain rates, while more rapid indentation rates could partially or even completely suppress the appearance of serrations. Our nanoindentation test on Be-bearing Zr-based BMG also shows a similar result [40]. The possible explanations for this rate dependency are as follows: at low strain rates single, isolated shear bands operate, whereas at high strain rates there are many shear bands operating within the specimen at the same instant. If the number of shear bands is sufficiently high, the contribution of an individual shear band to the total strain is indiscernible and, consequently, 
fewer serrations in $p-h$ curves can be observed. Wei et al. [73] recently performed an indentation study on seven BMG systems and found that the strain rate-dependent serrated behavior is not universal but strongly materialspecific, and it depends strongly on the homologous test temperature of $T / T_{\mathrm{g}}$ ( $T$ is the room temperature; $T_{\mathrm{g}}$ is the glass transition temperature). They observed that prominent serrated flow only occurs at low strain rates for the alloys with medium $T_{\mathrm{g}}$, whereas no distinct serrated flow was found in the materials with quite low or high $T_{\mathrm{g}}$. Obviously, further study is urgently required to reveal the physical origin of the serrated plastic flow and its rate dependency.

Another important issue associated with the indentation deformation behavior of BMGs is the pile-up phenomenon. This is actually a common feature of the indentation deformation behavior for most BMGs. The emergence of pile-up in BMG during indentation is due to the weakening or completely vanished work-hardening ability of the material. Although the main mechanism of pile-up seems to be at hand, how to evaluate hardness and the elastic-plastic constitutive response of BMGs with pile-up from the indentation testing data is still ongoing. In this context, a new modified expanding cavity model for characterizing the indentation behavior for pressuresensitive and work-hardening BMGs with pile-up was recently developed by Ai and Dai [71].

\subsection{Correlations among mechanical properties}

After years of study, vast amounts of experimental data of mechanical properties of BMGs are available. Interestingly, several striking correlations among mechanical properties of BMGs have been uncovered recently. Lewandoski et al. [15] found that there is a clear correlation between fracture toughness with the bulk-shear modulus ratio $(K / G)$ of BMGs, and metallic glasses with high $K / G$ show higher fracture toughness. Furthermore, Ashby and Greer [12] and $\mathrm{Xi}$ et al. [28] found that the fracture toughness of BMGs is highly correlated with the fracture process size $\left(w=k_{c}{ }^{2} / \pi \sigma_{y}{ }^{2}\right)$. If $w$ is much smaller than the sample size, fast fracture follows, while brittle failure is not expected if $w$ is greater than the sample size. Wang [74] made a relatively comprehensive survey on the existing data of bulk metallic glasses and found that the elastic moduli of BMG have correlations with the glass transition temperature $\left(T_{\mathrm{g}}\right)$, melting temperature, and the liquid fragility $\left(m=\left(d \log \eta / d \log \left(T_{\mathrm{g}} / T\right)\right), \eta\right.$ is the viscosity of liquid). After examining the available data of about 30 BMGs, Johnson and Samwer [75] demonstrated that BMGs bear a universal plastic yielding criterion with a $\left(T / T_{\mathrm{g}}\right)^{2 / 3}$ temperature dependence. Nieh et al. [76] found that the compression/tension or shear yield strength of BMGs at the ambient temperature $\left(T_{0}\right)$ can be completely determined by $T_{\mathrm{g}}$ and the molar volume $\left(V_{\mathrm{M}}\right)$ through a unified combination $\left(T_{\mathrm{g}}-T_{0}\right) / V_{\mathrm{M}}$. Very recently, we found [77] that there is an intrinsic compound correlation

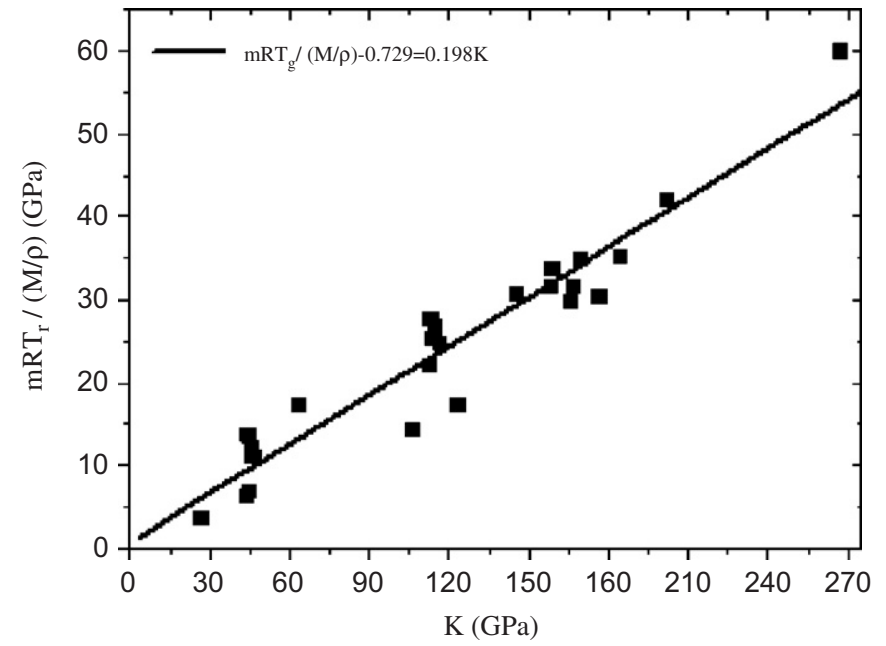

Fig. 8. Correlation between $m R T_{\mathrm{g}} /(M / \rho)$ and bulk modulus $K$ in metallic glasses [77].

between the bulk modulus $K$ of BMG and the fragility $m$ of its liquid, as shown in Fig. 8. Furthermore, we found that the correlation between the fragility $m$ and the bulk-shear modulus ratio $K / G$ follows a universal equation, i.e., $m=a(K / G)+b(T ; m)$, where $a$ is a material-independent constant and $b(T ; m)$ is a material-specific term. If the effect of temperature on elastic moduli is neglected or the measurement of elastic moduli is carried out at absolute zero, the Novikov-Sokolov's linear correlation between $m$ and $K / G$ is recovered [78]. It is noted that these correlations could assist in understanding the long-standing issue of glass transition, the nature of glass, and the glass-formation ability, which in turn may provide a new clue to discerning the susceptibility of shear banding in BMGs [79].

\section{Experimental observations of shear banding}

From the aforementioned discussions one can see that shear banding governs the macroscopic mechanical behavior of BMGs. Hence, extensive efforts have been made to explore the formation mechanism of shear bands in BMGs. Two hypotheses, namely the free volume softening and the thermal softening, were proposed to explain why shear bands are formed in BMGs [17,47-52]. However, the precise physical nature of the formation mechanism of shear bands in BMGs still remains unclear. To this end, we have made systematic experimental and analytical studies in recent years [24-26,53]. In this section, the main results of shear tests are outlined.

To investigate whether the strain rate and thermal softening exert an influence on the formation of shear bands in BMGs, both forced-shear and punch shear tests at the quasi-static strain rate $\left(\sim 1.5 \times 10^{-3} \mathrm{~s}^{-1}\right)$ and dynamic strain rate $\left(\sim 1.4 \times 10^{3} \mathrm{~s}^{-1}\right)$ were carried out $[24,25]$. Since the results obtained in these two types of tests are almost identical, only the forced-shear testing and its main results 

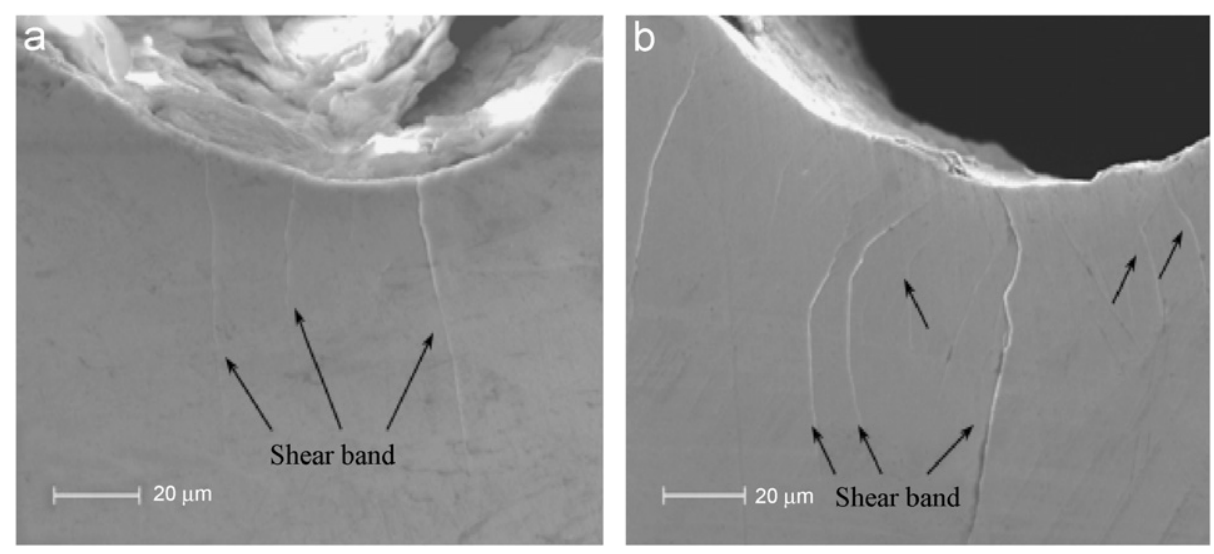

Fig. 9. The patterns of initiated shear bands: (a) quasi-static strain rates and (b) dynamic strain rates [24].

are discussed here [24]. The testing material is $\mathrm{Zr}_{41.2} \mathrm{Ti}_{13.8 \text { - }}$ $\mathrm{Cu}_{12.5} \mathrm{Ni}_{10} \mathrm{Be}_{22.5}$ bulk metallic glass (Vitreloy-1), which was fabricated by arc melting the pure elements together under a purified Ar atmosphere in ingots of the desired composition [26]. The quasi-static tests were conducted with a Microtest-2000 (GATAN) instrument, and dynamic shear tests with a split Hopkinson pressure bar. In order to conduct shear tests, the specimens were specially machined into a plate "hat-like" shape [24]. The shear stress-strain curves exhibit a "quasi-brittle" behavior and little macroscopic plastic flow, just like the tension case, see Fig. 1. Furthermore, the shear strength of the tested material displays no strain rate dependency. However, the formation of shear bands and the fracture surface pattern are strongly dependent on the strain rate. Fig. 9 presents the formed patterns of shear bands at two different strain rates. It can be readily seen from the figure that the number of shear bands is higher at dynamic strain rates than that at quasi-static strain rates. This rate dependency was also observed by Mukai et al. [22] in their quasi-static and dynamic tension tests. The fracture surface patterns for quasi-static and dynamic strain rates are shown in Fig. 10. For both cases, the fracture surface can be divided into three zones along the shear fracture direction: slip zone, propagation zone, and unstable fracture zone. However, their features of these three zones at dynamic strain rates are quite different from those at quasi-static strain rates. The most striking feature of the fracture surface at dynamic strain rates is that numerous liquid droplets and melted belts can be clearly observed in zone-II on the fracture surface, see Fig. 10b. This observation implies that adiabatic heating may exert certain influence on the shear fracture in BMGs. It is noted, however, that the melted liquid droplets cannot be observed in the fracture initiation zone. This means that the adiabatic heating softening may not be very pronounced in triggering the formation of shear bands in BMGs.

In addition, some other investigators observed nm-scale voids inside shear bands in metallic glasses with TEM examination [80,81]. It is thought that the formation of $\mathrm{nm}$-scale voids in shear bands should be attributed to local free volume coalescence. If so, this means that free volume softening may exert a certain influence on the formation of shear bands in BMGs. Therefore, the available experimental observations demonstrated that thermal softening, free volume softening, and strain rate are all possible factors affecting the shear band formation in BMGs. However, at the present stage, we still do not know as to how these factors affect the shear formation and also which one plays a dominant role. To clarify the formation mechanism of shear bands in BMGs, some quantitative theoretical analysis is urgently required.

\section{Mechanics of shear banding}

\subsection{Governing equations}

Consider a bulk metallic glass under one-dimensional dynamic simple shear, e.g., deformation can only occur in the direction of $x$-axis direction but there may be a gradient along the $y$-axis, which is shown in Fig. 11. Then, the governing equations for such a simple shear deformation involving both free volume softening and thermal softening are given by [53]

$\tau=f(\gamma, \dot{\gamma}, \theta, \xi)$,

$\frac{\partial^{2} \tau}{\partial y^{2}}=\rho \frac{\partial^{2} \gamma}{\partial t^{2}}$,

$\frac{\partial \theta}{\partial t}=\kappa \frac{\partial^{2} \theta}{\partial y^{2}}+\frac{K \tau}{\rho C_{v}} \frac{\partial \gamma}{\partial t}$

$\frac{\partial \xi}{\partial t}=D \frac{\partial^{2} \xi}{\partial y^{2}}+G(\xi, \theta, \tau)$,

where Eqs. (1)-(4) are the constitutive equation, the momentum equation, the energy equation, and the diffusion-production equation of the free volume concentration, respectively. Noticeably, the addition of Eq. (4) to the system of equations implies the consent to the 
a

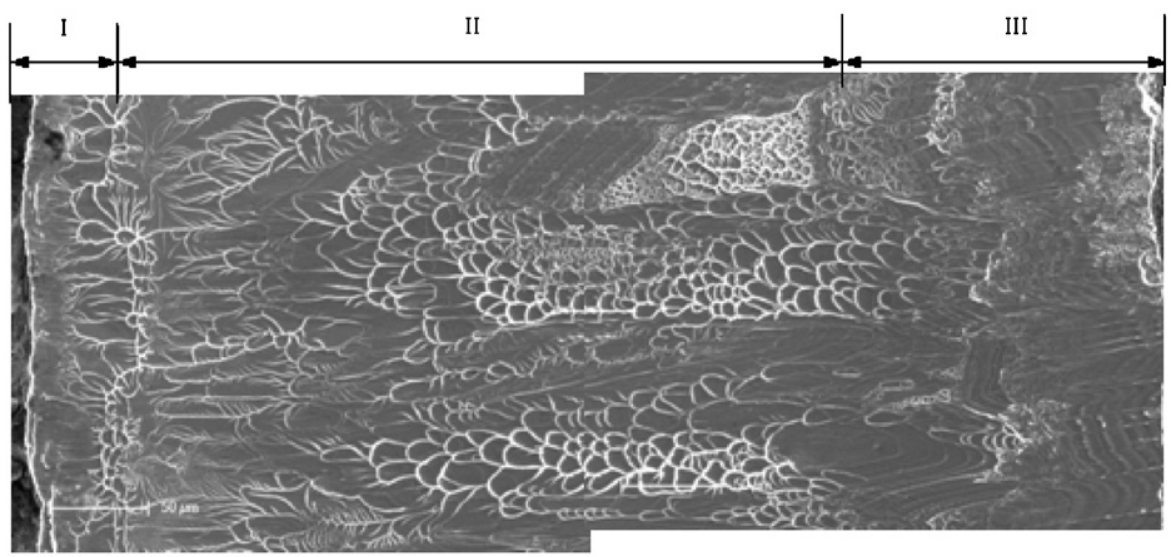

b
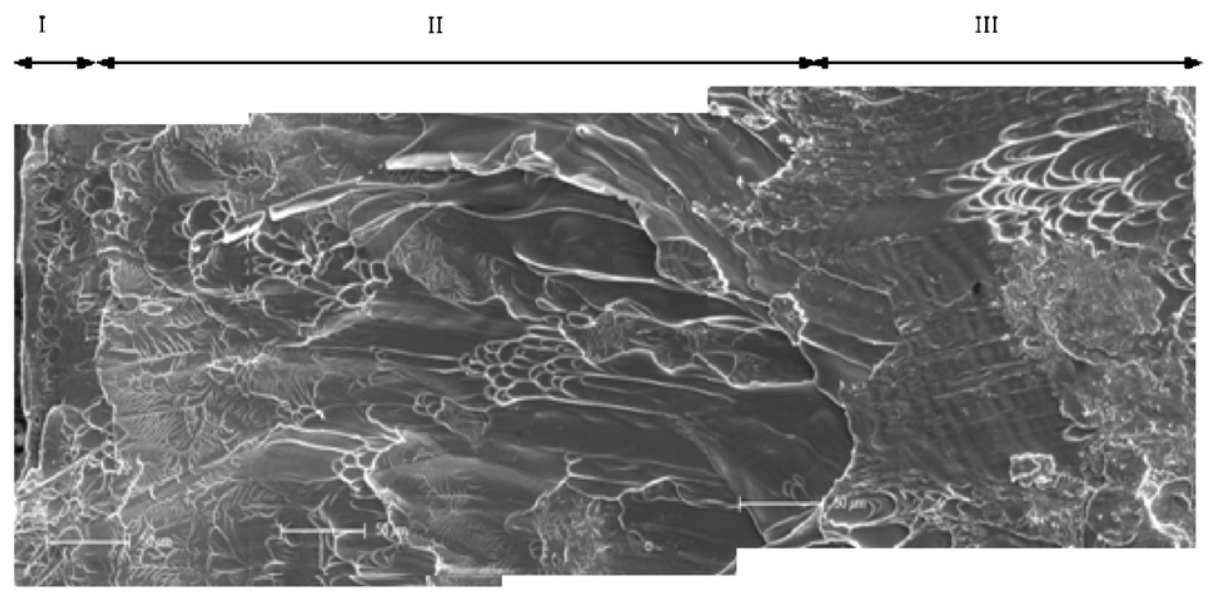

Fig. 10. Fracture surface patterns: (a) quasi-static case and (b) dynamic case [24].

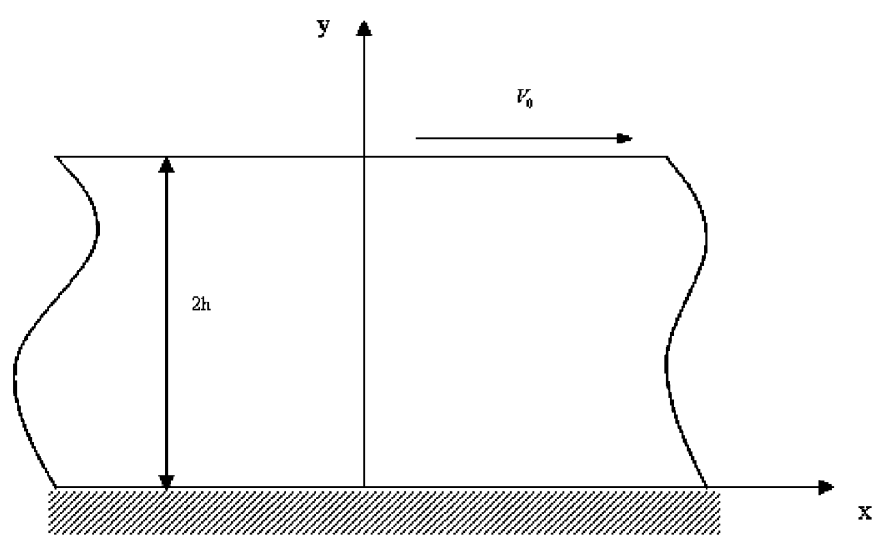

Fig. 11. Simple shearing of a BMG block.

shear-induced dilatation in BMGs, for which, actually, further validation is still required. In these equations, $\tau$ is the shear stress, $\gamma$ the shear strain, $\dot{\gamma}$ the shear strain rate, $\rho$ the density, $\theta$ the temperature, $C_{v}$ the specific heat, $\kappa=$ $\lambda / \rho C_{v}$ the thermal diffusion coefficient, $\lambda$ the thermal conductivity, $K$ the Taylor-Quinney coefficient $(K \cong 0.9), \xi$ the free volume concentration, and $D$ the diffusion coefficient of the free volume concentration. While $G(\xi, \theta, \tau)$ in Eq. (4) is the net generation rate function of the free volume concentration [17,50].

Since both free volume softening and thermal softening are included in the system characterized by Eqs. (1)-(4), this is a coupling problem. If neglecting the free volume softening, i.e., removing $\xi$ from Eq. (1) and deleting Eq. (4) from the governing system, the coupling problem reduces to the classical thermo-plastic shear instability studied previously in details [82]. Later this is referred to as the $T$-instability problem. If only free volume softening is considered, i.e., removing Eq. (3) from the governing system, the system reduces to the free volume softening governed or $V$-instability problem. If further confining to the quasi-static case, i.e. ignoring momentum equation (2), the problem is reduced to the case investigated previously by Huang et al. [50]. Obviously, in order to clarify the two mechanisms, namely thermal softening and free volume creation, one must straightforwardly tackle coupling deformation.

To study the coupling shear instability, we define several variables based on homogeneous deformation as follows: strain hardening $Q_{0}=(\partial \tau / \partial \gamma)_{0}$, strain rate hardening 
$R_{0}=(\partial \tau / \partial \dot{\gamma})_{0}$, thermal softening $P_{0}=-(\partial \tau / \partial \theta)_{0}$, free volume softening $F_{0}=-(\partial \tau / \partial \xi)_{0}$, and the three free volume creation-associated variables $G_{\xi}=\partial G / \partial \xi, G_{\theta}=$ $\partial G / \partial \theta$, and $G_{\tau}=\partial G / \partial \tau$.

\subsection{Linear instability analysis}

Linear perturbation to the system of governing equations (1)-(4) gives the following characteristic equation for eigenvalue $\alpha$ [43]. Importantly, $\alpha$ is the reciprocal of characteristic time and describes the exponential growth (when $\alpha$ has a positive real part) or decay (all real parts of $\alpha$ are negative) of perturbations $\mathrm{e}^{\alpha t+\mathrm{i} k y}$, where $k$ is the wave number:

$a_{4} \alpha^{4}+a_{3} \alpha^{3}+a_{2} \alpha^{2}+a_{1} \alpha+a_{0}=0$.

According to the Routh-Hurwitz theorem (see, for example, Korn and Korn [83]), if there are sign changes in the elements in the first column of Routh array, such as the coefficient $a_{4}$ is positive and $a_{0}<0$, then at least one of the roots of the characteristic equation (5) has a positive real part $\alpha>0$, indicating the occurrence of unstable deformation. So, based on this characteristic equation, three types of instability modes, i.e., the coupling instability, $T$-instability, and $V$-instability, will be discussed as follows.

\subsubsection{Coupling instability}

In this case, both free volume softening and thermal softening are included in the governing system and the coefficients of the characteristic equation (5) are:

$a_{4}=1$,

$a_{3}=\left[\beta+\rho C_{v}\left(D k^{2}-G_{\xi}\right)+\rho C_{v} G_{\tau} F_{0}\right] / \rho C_{v}$,

$a_{2}=\left[k^{2} \omega+\rho \beta\left(D k^{2}-G_{\xi}\right)+k^{2} \rho \lambda G_{\tau} F_{0}+K \dot{\gamma}_{0} \rho G_{\theta} F_{0}\right] / \rho^{2} C_{v}$,

$a_{1}=k^{2}\left[\omega\left(D k^{2}-G_{\xi}\right)+\lambda Q_{0} k^{2}-K \tau_{0} G_{\theta} F_{0}\right] / \rho^{2} C_{v}$,

$a_{0}=k^{4} \lambda Q_{0}\left(D k^{2}-G_{\xi}\right) / \rho^{2} C_{v}$,

$\beta=K \dot{\gamma}_{0} P_{0}+\lambda k^{2}+R_{0} C_{v} k^{2}, \quad \omega=\lambda R_{0} k^{2}+\rho C_{v} Q_{0}-K \tau_{0} P_{0}$.

At the short wave limit $(k \rightarrow \infty), \alpha$ has a negative solution only,

$\alpha=-Q_{0} / R_{0}$.

So, homogeneous deformation is stable for the short wave case. At the long wave limit $(k \rightarrow 0)$, the instability condition is $G_{\xi}>G_{\tau} F_{0}+K P_{0} \dot{\gamma}_{0} / \rho C_{v}$ or $G_{\xi} P_{0}>G_{\theta} F_{0}$.

In general, i.e., wave number $k$ is neither very small nor very large; according to Eqs. (6)-(11), an easy way to instability is

$G_{\xi}>D k^{2}$, where $G_{\xi}=\partial G / \partial \xi$ stands for the compound creation rate of free volume and $D k^{2}$ for the diffusion rate of free volume. The instability condition (13) means that if the compound creation rate of free volume exceeds the free volume diffusion rate, the uniform deformation would become unstable, namely instability would occur. So, essentially, this instability criterion is of rate competition and there is always a wave number threshold, beyond which the perturbations with shorter wave numbers will grow exponentially.

In the following, let us compare this coupling instability with $T$ - and $V$-instabilities, respectively.

\subsubsection{T-instability}

In this case, only thermal softening is considered and the coefficients of the characteristic equation (5) become

$a_{4}=0$,

$a_{3}=1$,

$a_{2}=\beta / \rho C_{v}=\left[K \dot{\gamma}_{0} P_{0}+\lambda k^{2}+R_{0} C_{v} k^{2}\right] / \rho C_{v}$,

$a_{1}=\omega k^{2} / \rho^{2} C_{v}=k^{2}\left[\lambda R_{0} k^{2}+\rho C_{v} Q_{0}-K \tau_{0} P_{0}\right] / \rho^{2} C_{v}$,

$a_{0}=\lambda Q_{0} k^{4} / \rho^{2} C_{v}$.

At the short wave limit $(k \rightarrow \infty)$, the above $\alpha$ has negative root only

$\alpha=-Q_{0} / R_{0}$

and homogeneous deformation remains stable. At the long wave limit $(k \rightarrow 0)$, the deformation also keeps stable because $\alpha$ takes negative value only, i.e., $\alpha=-K P_{0} \dot{\gamma}_{0} / \rho C_{v}$. In between, there exists the fastest growth wave mode, showing instability. The condition for this dominant unstable mode can be simply written as

$B=\frac{K P_{0} \tau_{0}}{\rho C_{v} Q_{0}}>1$.

This instability condition was obtained previously in [82] and $B$ is a dimensionless number reflecting the competition between thermal softening $P_{0}$ and strain hardening $Q_{0}$. So essentially, this instability criterion is a type of energy condition. This instability condition tells us that if thermal softening overcomes strain hardening, uniform deformation will become unstable.

\subsubsection{V-instability}

In this case, only free volume softening is considered and the coefficients of the characteristic equation (5) become

$a_{4}=0$,

$a_{3}=1$,

$a_{2}=\left(D k^{2}-G_{\xi}\right)+R_{0} k^{2} / \rho+G_{\tau} F_{0}$, 
$a_{1}=k^{2}\left[Q_{0}+R_{0}\left(D k^{2}-G_{\xi}\right)\right] / \rho$,

$a_{0}=k^{2} Q_{0}\left(D k^{2}-G_{\xi}\right) / \rho$.

Firstly, examine two extreme cases. At the short wave limit $(k \rightarrow \infty), \alpha$, again, has a negative solution only, $(k \rightarrow$ $\infty)$

$\alpha=-Q_{0} / R_{0}$.

Hence, homogeneous deformation is stable in this case. At the long wave limit $(k \rightarrow 0)$, uniform deformation may become unstable provided $G_{\xi}>G_{\tau} F_{0}$.

Generally, homogeneous deformation becomes unstable if the following condition is met:

$G_{\xi}>D k^{2}$.

It is noted that this instability condition was also obtained previously by Huang et al. [50]. However, the interesting thing is that this condition for $V$-instability is the same as that in the coupling case, Eq. (13). According to this fact, the free volume creation seems to play a dominant role in controlling the formation of shear bands in BMGs.

Above all, the aforementioned three deformation modes remain stable in the short wave limit case, and $\alpha$ takes an identical value $\alpha=-Q_{0} / R_{0}$. The reciprocal of this eigenvalue $\alpha$ defines a characteristic time scale

$t_{R Q}=\frac{1}{|\alpha|}=\frac{R_{0}}{Q_{0}}$,

which corresponds to the characteristic time for the deformed material to relax to the stable state. Hence, the relaxation time $t_{R Q}$ is an intrinsic time scale for the material under consideration. In particular, all the variables relevant to softening, such as thermal softening $P_{0}$, free volume softening $F_{0}$ as well as compound creation of free volume $G_{\xi}$, do not appear in the characteristic equation at the short wave limit.

\subsection{Growth time of shear instability}

As soon as instability occurs, how fast it grows is the other key issue for shear banding. Again, we examine the problem from perturbation approximation to get the estimation of growth time separately.

For $T$-instability, the approximate growth time can be estimated as [82]

$t_{\theta} \approx 1 /|\alpha|=\frac{R_{0} / Q_{0}}{\left(K P_{0} \tau_{0}\right) /\left(\rho C_{v} Q_{0}\right)-1}=\frac{t_{R Q}}{B-1}$.

For $V$-instability, the instability growth time would be

$t_{V} \approx 1 /|\alpha|=1 / G_{\xi}$.

For the coupling instability case, the instability growth time is given by [53]

$t_{\mathrm{c}}=\frac{2 \rho C_{v} R_{0}}{K \tau_{0} P_{0}-\rho C_{v} Q_{0}+\rho C_{v} R_{0} G_{\xi}+\sqrt{\left(K \tau_{0} P_{0}-\rho C_{v} Q_{0}+\rho C_{v} R_{0} G_{\xi}\right)^{2}}}$.
It can readily be seen from Eq. (31) that strain hardening and strain rate hardening retard the perturbation growth, whereas thermal softening $P_{0}$ and compound free volume creation $G_{\xi}$ make the instability grow faster. This is quite different from that for the static case discussed in [50]. As for the relative importance of the free volume softening and the thermal softening, the compound free volume creation $G_{\xi}$ appears to govern the instability growth time around $B \approx 1$. This can be seen more clearly, when the instability growth time given by Eq. (31) is converted into the following two simple expressions.

It is noted that, if the compound free volume creation is dominant, i.e., $K \tau_{0} P_{0}-\rho C_{v} Q_{0}<\rho C_{v} R_{0} G_{\xi}$, then the instability time reduces to the $V$-instability case,

$t_{\mathrm{c}}=t_{V}=\frac{1}{G_{\xi}}$.

If thermal softening is dominant, i.e., $K \tau_{0} P_{0}-\rho C_{v} Q_{0}>\rho C_{v} R_{0} G_{\xi}$, then the instability time is the same as that in the $T$-instability case,

$t_{\mathrm{c}}=t_{\theta}=\frac{R_{0}^{*}}{\dot{\gamma}_{0}} \frac{\rho C_{v}}{K \tau_{0} P_{0}-\rho C_{v} Q_{0}}=\frac{t_{R Q}}{B-1}$,

where $R_{0}^{*}=\partial \tau / \partial \ln \left(\dot{\gamma} / \dot{\gamma}_{0}\right)$ is the strain rate sensitivity coefficient of materials.

\subsection{Width of shear bands}

Since most experimental observations are post mortem patterns of shear bands, to get some theoretical estimation on the mature shear band width and to make comparison of the estimation to the observations are helpful. Based on the basic equations (1)-(4), the late-stage steady equations and band-like solutions for the $T$-instability mode and $V$-instability mode can be obtained. For $T$-banding, the solution is

$y=\sqrt{\frac{\kappa}{2 K \tau}} \int_{0}^{\theta_{m}} \frac{\mathrm{d} \varsigma}{\sqrt{\int_{\varsigma}^{\theta_{m}} g(\tau, \eta) \mathrm{d} \eta}}$.

For $V$-banding, the solution is

$y=\sqrt{\frac{D}{2}} \int_{\varsigma}^{\varsigma_{m}} \frac{\mathrm{d} \varsigma}{\sqrt{\int_{\zeta}^{\varsigma_{m}} G(\eta, \theta, \tau) \mathrm{d} \eta}}$,

where $y$ is the coordinate vertical to the band and subscript $m$ denotes the value at the center of the band. Clearly, there is a similarity between thermal and free volume diffusions in the steady solutions. However, the difference is distinctive: their integrands are different. One is the strain rate (namely external time scale), the other is the free volume creation $G$, namely an intrinsic one. Then a simplified but explicit expression of the shear band width in the two cases could be written as

$\delta_{T} \sim \sqrt{\kappa \frac{\gamma^{*}}{\dot{\gamma}^{*}} \frac{\rho C_{v} \theta^{*}}{K \tau^{*} \gamma^{*}}} \sim \sqrt{\kappa \frac{\gamma^{*}}{\dot{\gamma}^{*}}}$ 
because of $\left(\rho C_{v} \theta^{*} / K \tau^{*} \gamma^{*}\right) \sim(1 / B)=\left(\rho C_{v} Q_{0} / K P_{0} \tau_{0}\right) \sim 1$, and

$\delta_{V} \sim \sqrt{D \frac{\xi^{*}}{G^{*}}}$

where $*$ denotes the values within the band. Again, take $\mathrm{Zr}_{41.2} \mathrm{Ti}_{13.8} \mathrm{Cu}_{12.5} \mathrm{Ni}_{10} \mathrm{Be}_{22.5}$ bulk metallic glass as a model material $[27,50]$. Roughly, we take the following approximate estimations for the relevant parameters for the $\dot{\gamma}_{0} \sim 10^{3} \mathrm{~s}^{-1}$ case: $\kappa \sim 10^{-6} \mathrm{~ms}^{-2}, \quad D \sim 10^{-16} \mathrm{~ms}^{-2}, \gamma^{*} \sim 10^{2}$, $\dot{\gamma}^{*} \sim 10^{5} \mathrm{~s}^{-1}, \xi^{*} \sim 10^{-2}$, and $G^{*} \sim 10^{1} \mathrm{~s}^{-1}$. Accordingly, we have $\delta_{T} \sim 10 \mu \mathrm{m}$ and $\delta_{V} \sim \mathrm{nm}$, respectively. It is noted that the theoretical estimation of the width of shear bands for $V$-shear banding is consistent with the available experimental observations in BMGs, where the band width ranges from several to tens of nanometers $[81,84]$. So, it seems that the mature feature of shear bands in BMGs looks more like the $V$-shear band and thermal effects just assist to accelerate the instability growth as shown in Eq. (36). However, more complete theoretical and numerical investigations are needed to clarify the whole process of shear band evolution. This work is underway and we will make a further report on this issue.

\section{Conclusions}

In this paper, some basic mechanical behaviors of BMGs are discussed. From the discussions, one can find that these mechanical behaviors of BMGs are mainly resulted from the formation and operation of shear bands in BMGs. Therefore, relevant mechanics of shear banding were investigated in the paper. Our theoretical analysis demonstrates that: (1) For V-instability governed by free volume creation, the deformation will become unstable if the compound volume creation rate exceeds the free volume diffusion rate, i.e. $G_{\xi}>D k^{2}$. (2) For $T$-instability governed by thermal softening, the deformation becomes unstable provided $B=\left(K P_{0} \tau_{0} / \rho C_{v} Q_{0}\right)>1$. (3) For the deformation with coupled thermal effects and free volume creation, the compound free volume creation and thermal softening can jointly initiate and accelerate the instability growth. However, the compound free volume creation $G_{\xi}$ appears to govern the instability growth when $B \approx 1$. (4) According to the late-stage steady solutions, the shear band width for $V$-instability and T-instability can be estimated by $\delta_{V} \approx$ $\sqrt{D\left(\xi^{*} / G^{*}\right)} \sim$ nm and $\delta_{T} \approx \sqrt{\kappa\left(\gamma^{*} / \dot{\gamma}^{*}\right)}$, respectively. The observed post mortem shear band width of nanometers in BMGs looks more like the former governed by free volume creation.

\section{Acknowledgments}

The authors gratefully acknowledge the support of the National Natural Science Foundation of China under Grants nos. 10432050, 10721202, 10725211, and 10472119 and the innovation programs of Chinese Academy of
Sciences (KJCX-SW-L08 and KJCX2-YW-M04). They also acknowledge the State Key Laboratory of Scientific and Engineering Computing, Chinese Academy of Sciences, for providing parallel computing resources.

\section{References}

[1] Turnbull D, Cech RE. Microscopic observation of the solidification of small metal droplets. J Appl Phys 1950;21:804-10.

[2] Klement WJ, Willens RH, Duwez P. Non-crystalline structure in solidified gold-silicon alloys. Nature 1960;187:869.

[3] Chen HS, Turnbull D. Formation, stability, and structure of palladium-silicon based alloy glass. Acta Metall 1969;17:1021-31.

[4] Chen HS. Thermodynamic consideration on the formation and stability of metallic glasses. Acta Metall 1974;22:1505-11.

[5] Kui HW, Greer AL, Turnbull D. Formation of bulk metallic glass by fluxing. Appl Phys Lett 1984;45:615-6.

[6] Inoue A, Kato A, Zhang T, Kim SG, Masumoto T. $\mathrm{Mg}-\mathrm{Cu}-\mathrm{Y}$ amorphous alloys with high strengths produced by a metallic mold casting method. Mater Trans JIM 1991;32:609-16.

[7] Peker A, Johnson WL. A highly processable metallic glass $\mathrm{Zr}_{41.2} \mathrm{Ti}_{13.8} \mathrm{Cu}_{12.5} \mathrm{Ni}_{10.0} \mathrm{Be}_{22.5}$. Appl Phys Lett 1993;63:2342-4.

[8] Greer AL. Metallic glasses. Science 1995;267:1947-53.

[9] Johnson WL. Bulk glass-forming metallic alloys: science and technology. Mater Res Bull 1999;24:42-56.

[10] Inoue A. Stabilization of metallic supercooled liquid and bulk amorphous alloys. Acta Mater 2000;48:279-306.

[11] Wang WH, Dong C, Shek CH. Bulk metallic glasses. Mater Sci Eng R 2004;44:45-89.

[12] Ashby MF, Greer AL. Bulk metallic glasses as structural materials. Scr Mater 2006;54:321-6.

[13] Eckert J, Das J, Pauly S, Duhamel C. Mechanical properties of bulk metallic glasses and composites. J Mater Res 2007;22:285-301.

[14] Inoue A, Shen BL, Chang CT. Super-high strength of over $4000 \mathrm{MPa}$ for Fe-based glassy alloys in $\left[\left(\mathrm{Fe}_{1-x} \mathrm{Co}_{x}\right)_{0.75} \mathrm{~B}_{0.2} \mathrm{Si}_{0.05}\right]_{96} \mathrm{Nb}_{4}$ System. Acta Mater 2004;52:4093-9.

[15] Lewandowski JJ, Wang WH, Greer AL. Intrinsic plasticity or brittleness of metallic glasses. Philos Mag Lett 2005;85:77-87.

[16] Fu XY, Rigney DA, Falk ML. Sliding and deformation of metallic glass: experiments and MD simulations. J Non-Crys Solids 2003;317:206-214I.

[17] Spaepen F. A microscopic mechanism for steady state inhomogeneous flow in metallic glasses. Acta Metall 1977;25:407-15.

[18] Pampillo CA. Flow and fracture in amorphous alloys. J Mater Sci 1975;10:1194-227.

[19] Hufnagel TC, El-Deiry P, Vinci RP. Development of shear band structure during deformation of $\mathrm{Zr}_{57} \mathrm{Ti}_{5} \mathrm{Cu}_{20} \mathrm{Ni}_{8} \mathrm{Al}_{10}$ bulk metallic glass. Scr Mater 2000;43:1071-5.

[20] Yang B, Morrison ML, Liaw PK, Buchanan RA, Wang G, Liu CT, et al. Dynamic evolution of nanoscale shear bands in a bulk metallic glass. Appl Phys Lett 2005;86:14904-13.

[21] Zhang ZF, Eckert J, Schultz L. Difference in compressive and tensile fracture mechanisms of $\mathrm{Zr}_{59} \mathrm{Cu}_{20} \mathrm{Al}_{10} \mathrm{Ni}_{8} \mathrm{Ti}_{3}$ bulk metallic glass. Acta Mater 2003;51:1167-79.

[22] Mukai T, Nieh TG, Kawamura Y, Inoue A, Higashi K. Dynamic response of a $\mathrm{Pd}_{40} \mathrm{Ni}_{40} \mathrm{P}_{20}$ bulk metallic glass in tension. Scr Mater 2002;46:43-7.

[23] Wang G, Shen J, Sun JF, Lu ZP, Stachurski ZH, Zhou BD. Tensile fracture characteristic and deformation behavior of a $\mathrm{Zr}$-based bulk metallic glass at high temperatures. Intermetallics 2005;13:642-8.

[24] Liu LF, Dai LH, Bai YL, Wei BC. Initiation and propagation of shear bands in Zr-based bulk metallic glass under quasi-static and dynamic shear loadings. J Non-Crystall Solids 2005;351:3259-70.

[25] Liu LF, Dai LH, Bai YL, Wei BC, Eckert J. Characterization of ratedependent shear behavior of Zr-based bulk metallic glass using shear punch testing. J Mater Res 2006;21:153-60. 
[26] Liu LF, Dai LH, Bai YL, Wei BC, Eckert J. Behavior of multiple shear bands in Zr-based bulk metallic glass. Mater Chem Phys 2005;93:174-7.

[27] Lu J, Ravichandran G, Johnson WL. Deformation behavior of the $\mathrm{Zr}_{41.2} \mathrm{Ti}_{13.8} \mathrm{Cu}_{12.5} \mathrm{Ni}_{10} \mathrm{Be}_{22.5}$ bulk metallic glass over a wide range of strain-rates and temperatures. Acta Mater 2003;51: $3429-43$

[28] Xi XK, Zhao DQ, Pan MX, Wang WH, Wu Y, Lewandowski JJ. Fracture of brittle metallic glasses: brittleness or plasticity. Phys Rev Lett 2005;94:1255101-4.

[29] Xi XK, Zhao DQ, Pan MX, Wang WH, Wu Y, Lewandowski JJ. Periodic corrugation on dynamic fracture surface in brittle bulk metallic glass. Appl Phys Lett 2006;89:1819111-3.

[30] Wang G, Wang YT, Liu YH, Pan MX, Zhao DQ, Wang WH. Evolution of nanoscale morphology on fracture surface of brittle metallic glass. Appl Phys Lett 2006;89:1219091-3.

[31] Shen J, Liang WZ, Sun JF. Formation of nanowaves in compressive fracture of a less-brittle bulk metallic glass. Appl Phys Lett 2006; 89:121908

[32] Zhang ZF, Wu FF, Gao W, Tan J, Wang ZG, Stoica M, et al. Wavy cleavage fracture of bulk metallic glass. Appl Phys Lett 2006; 89:2519171-3.

[33] Pan DG, Zhang HF, Wang AM, Wang ZG, Hu ZQ. Fracture instability in Mg-based bulk metallic glasses. J Alloys Compd 2007;438:145-9.

[34] Kimura H, Masumoto T. A model of the mechanics of serrated flow in an amorphous alloy. Acta Metall 1983;31:231-40.

[35] Wright WJ, Saha R, Nix WD. Deformation mechanisms of the $\mathrm{Zr}_{40} \mathrm{Ti}_{14} \mathrm{Ni}_{10} \mathrm{Cu}_{12} \mathrm{Be}_{24}$ bulk metallic glass. Mater Trans JIM 2001;42: 642-9.

[36] Schuh CA, Neih TG. A nanoindentation study of serrated flow in bulk metallic glasses. Acta Mater 2003;51:87-99.

[37] Jiang WH, Atzmon M. Rate dependence of serrated flow in a metallic glass. J Mater Res 2003;19:755-7.

[38] Greer AL, Castellero, Madge SV, Walker IT, Wilde JR. Nanoindentation studies of shear banding in fully amorphous and partly devitrified metallic alloys. Mater Sci Eng 2004;A375-A377: $1182-5$.

[39] Liu LF, Dai LH, Bai YL, Wei BC. Strain rate-dependent compressive deformation behavior of Nd-based bulk metallic glass. Intermetallics 2005;13:827-32.

[40] Dai LH, Liu LF, Yan M, Wei BC, Eckert J. Serrated plastic flow in a Zr-based bulk metallic glass during nanoindentation. Chin Phys Lett 2004;21:1593-5.

[41] Wei BC, Zhang LC, Zhang TH, Xing DM, Das J, Eckert J. Strain rate dependence of plastic flow in Ce-based bulk metallic glass during nanoindentation. J Mater Res 2007;22:1-5.

[42] Yang B, Nieh TG. Effect of the nanoindentation rate on the shear band formation in an Au-based bulk metallic glass. Acta Mater 2007;55:295-3000.

[43] Bai YL, Dodd B. Adiabatic shear localization. Oxford: Pergamon Press; 1992.

[44] Meyers MA. Dynamic behavior of materials. New York: Wiley; 1994.

[45] Wright TW. The physics and mathematics of adiabatic shear bands. Cambridge: Cambridge University Press; 2002.

[46] Batra RC, Wei ZG. Shear band spacing in thermoviscoplastic materials. Int J Impact Eng 2006;32:947-67.

[47] Argon AS. Plastic deformation in metallic glasses. Acta Metall 1979;27:47-58.

[48] Steif PS, Spaepen F, Huchinson JW. Strain localization in amorphous metals. Acta Metall 1982;30:447-55.

[49] Wright W, Hufangel TC, Nix WD. Free volume coalescence and void formation in shear bands in metallic glass. J Appl Phys 2003;93: $1432-7$.

[50] Huang R, Suo Z, Prevost JH, Nix WD. Inhomogeneous deformation in metallic glasses. J Mech Phys Solids 2002;50:1011-27.

[51] Leamy HJ, Chen HS, Wang TT. Plastic flow and fracture of metallic glass. Metall Trans 1972;3:699-708.
[52] Liu CT, Heatherly L, Easton DS, Carmichael CA, Schneibel JH, $\mathrm{Chen} \mathrm{CH}$. Test environments and mechanical properties of $\mathrm{Zr}$-based bulk amorphous alloys. Metall Mater Trans 1998;A29:1811-20.

[53] Dai LH, Yan M, Liu LF, Bai YL. Adiabatic shear banding instability in bulk metallic glasses. Appl Phys Lett 2005;87:141916.

[54] Anand L, Su C. A theory for amorphous viscoplastic materials undergoing finite deformation, with application to metallic glasses. J Mech Phys Solids 2005;53:1362-96.

[55] Yang Q, Mota A, Ortiz M. A finite deformation constitutive model of bulk metallic glass plasticity. Comput Mech 2006;37:194-204.

[56] Bruck HA, Rosakis AJ, Johnson WL. The dynamic compressive behavior of beryllium bearing bulk metallic glass. J Mater Res 1996; 11:503-11.

[57] Hufanagel TC, Jiao T, Li Y, Xing LQ, Ramesh KT. Deformation and failure of $\mathrm{Zr}_{57} \mathrm{Ti}_{5} \mathrm{Cu}_{20} \mathrm{Ni}_{8} \mathrm{Al}_{10}$. J Mater Res 2002:1441-5.

[58] Maddin R, Masumoto T. The deformation of amorphous palladium$20 \%$ at silicon. Mater Sci Eng 1972;9:153-62.

[59] Conner RD, Dandliker RB, Scruggs V, Johnson WL. Dynamic deformation behavior of tungsten-fiber/metallic-glass matrix composites. Int J Impact Eng 2000;24:435-44.

[60] Martin M, Thadhani NN, Kockes L, Dowding R. Instrumented anvil-rod-impact testing of a bulk metallic glass composite for constitutive model validation. Scr Mater 2006;55:1019-22.

[61] Zhuang S, Lu J, Ravichandran G. Shock wave response of a zirconium-based bulk metallic glass and its composite. Appl Phys Lett 2002;80:4522-4.

[62] Tumeaure SJ, Winey JM, Gupta YM. Appl Phys Lett 2004;84: 1692-4.

[63] Fineberg J, Gross SP, Marder M, Swiney H. Instability in dynamic fracture. Phys Rev Lett 1991;67:457-60.

[64] Spaepen F, Turnbull D. A mechanism for the flow and fracture of metallic glasses. Scr Metall 1974;8:563-8.

[65] Wang G, Pan H, Ke F, Xia MF, Bai YL. Strain rate effect on the mechanical properties of amorphous copper. Chin Phys B 2008;17(1): 259-63.

[66] Wang G, Pan H, Ke F, Xia MF, Bai YL. A microscopic study of formation and mechanical properties of amorphous $\mathrm{Cu}$. J Beijing Univ Aeronaut Astronaut 2007;33(6):752-6.

[67] Wang G, Xu XH, Han YN, Han BS, Ke FJ, Wang WH. Ductile to brittle transition in dynamic fracture of a brittle bulk metallic glass. Phys Rev Lett, submitted for publication.

[68] Jiang MQ, Ling Z, Meng JX, Dai LH. Energy dissipation in fracture of bulk metallic glasses via inherent competition between local softening and quasi-cleavage. Phil Mag 2008;88(3):407-26.

[69] Oliver WC, Pharr GM. An improved technique for determining hardness and elastic modulus using load and displacement sensing indentation measurements. J Mater Res 1992;7:1564-83.

[70] Cheng YT, Cheng CM. Scaling, dimensional analysis, and indentation measurements. Mater Sci Eng R 2004;44:91-149.

[71] Ai K, Dai LH. A new modified expanding cavity model for characterizing the spherical indentation behavior of bulk metallic glass with pile-up. Scr Mater 2007;56:761-4.

[72] Robinson JM. Serrated flow in aluminum base alloy. Int Mater Rev 1994;39:217-27.

[73] Li WH, Wei BC, Zhang TH, Xing DM, Zhang LC, Wang YR. Study of serrated flow and plastic deformation in metallic glasses through instrumental indentation. Intermetallics 2007;15:706-10.

[74] Wang WH. Correlations between elastic moduli and properties in bulk metallic glasses. J Appl Phys 2006; 99:093506 $1-093506_{10}$.

[75] Johnson WL, Samwer K. A universal criterion for plastic yielding of metallic glasses with a $\left(T / T_{\mathrm{g}}\right)^{2 / 3}$ temperature dependence. Phys Rev Lett 2005;95:1955011-4.

[76] Yang B, Liu CT, Nieh TG. Unified equation for the strength of bulk metallic glasses. Appl Phys Lett 2006;88:2219111-4.

[77] Jiang MQ, Dai LH. An intrinsic correlation between fragility and bulk modulus in metallic glasses. Phys Rev B 2007; 76:054204 ${ }_{1}-054204_{7}$.

[78] Noviko VN, Sokolov AP. Poisson's ratio and the fragility of glassforming liquids. Nature 2004;431:961-3. 
[79] Dai LH, Jiang MQ. The fragility of liquid and its correlation with mechanical properties of glasses. Adv Mech 2007;37:346-60.

[80] Donovan PE, Stobbs WM. The structure of shear bands in metallic glasses. Acta Metall 1981;29:1419-36.

[81] Li J, Spaepan F, Hufnagel TC. Nanometer scale defects in shear bands in a metallic glass. Philos Mag 2002;A82:2623-30.
[82] Bai YL. Thermo-plastic instability in simple shear. J Mech Phys Solids 1982;30:195-207.

[83] Korn GA, Korn TM. Mathermatical handbook for scientists and engineers. New York: McGraw-Hill; 1961.

[84] Zhang Y, Greer AL. Thickness of shear bands in metallic glasses. Appl Phys Lett 2006;89:071907. 